\title{
3D molecular models of whole HIV-1 virions generated with cellPACK
}

\author{
Graham T. Johnson, *abc David S. Goodsell, ${ }^{\mathrm{C}}$ Ludovic Autin, ${ }^{\mathrm{C}}$ \\ Stefano Forli, ${ }^{C}$ Michel F. Sanner ${ }^{C}$ and Arthur J. Olson ${ }^{c}$
}

Received 19th February 2014, Accepted 19th March 2014

DOI: $10.1039 / c 4 f d 00017 j$

As knowledge of individual biological processes grows, it becomes increasingly useful to frame new findings within their larger biological contexts in order to generate new systems-scale hypotheses. This report highlights two major iterations of a whole virus model of HIV-1, generated with the cellPACK software. cellPACK integrates structural and systems biology data with packing algorithms to assemble comprehensive 3D models of cell-scale structures in molecular detail. This report describes the biological data, modeling parameters and cellPACK methods used to specify and construct editable models for HIV-1. Anticipating that cellPACK interfaces under development will enable researchers from diverse backgrounds to critique and improve the biological models, we discuss how cellPACK can be used as a framework to unify different types of data across all scales of biology.

\section{Introduction}

\section{Biological processes in their mesoscale context}

Multiscale biological models that span from atoms to cells can greatly improve our understanding of the mechanisms of health and disease. However, methods do not yet exist to observe, visualize, or model the mesoscale, the intermediate scale of $10^{-7}-10^{-8} \mathrm{~m}$ bridging molecular and cellular biology, in full molecular detail. To address this need, we have developed a software framework called cellPACK, which integrates multiple types of data across scales into comprehensive 3D spatial models. This report describes a cellPACK pipeline used to create increasingly rigorous molecular models of HIV-1 (Human Immunodeficiency Virus) from structural components and systems biology data.

Structural modeling of HIV-1 poses a timely challenge. HIV is a membraneenveloped lentivirus that approaches a cell in its complexity. The genome of HIV is packaged inside a protein capsid, which requires a sophisticated collection of

\footnotetext{
${ }^{a}$ University of California, San Francisco, CA 94143, USA. E-mail: graham.johnson@ucsf.edu ${ }^{b}$ California Institute for Quantitative Biosciences (QB3), QB3 MC: 2522, 1700 4th Street, Byers Hall, Suite 214, San Francisco, CA 94158-2330

'The Scripps Research Institute, 10550 North Torrey Pines Road, La Jolla, CA 92037, USA
} 
viral and cellular molecules to deliver it to new hosting cells, to initiate replication of the genome, and to repackage the necessary viral components for successful infection. ${ }^{1}$ NIGMS has recently created a series of Specialized Centers, bringing together dozens of research labs to characterize the structures and mechanisms across the entire lifecycle, and to identify rational targets for drug and vaccine design (http://www.nigms.nih.gov/Research/SpecificAreas/AIDSStructuralBiology/ Pages/HIVspecializedcenters.aspx).

The goal of cellPACK is to create rigorous structural models of HIV and its interactions with host cells, which frame our current knowledge on the scale of the entire virion, and in its cellular context in a consistent format. These models will impel hypothesis-driven research by integrating diverse data types, identifying gaps in knowledge, and exploring the ranges of models that are consistent with current knowledge. The level of complexity of HIV is ideally suited to push the current capabilities of cellPACK and to uncover the development needs of the open-source cellPACK project as it evolves to model larger systems.

\section{Methods to bridge from atoms to cells}

Experimental techniques for integrative structural biology are now linking molecular structure and supramolecular structure, for instance, to explore the structure of HIV glycoprotein multimers ${ }^{2}$ and HIV capsid. ${ }^{3,4}$ However, methods to observe, visualize or model the larger end of the mesoscale in atomic-resolution detail do not yet exist. To make the next step, from atoms to significant portions of cells, many sources of data can be used to synthesize a view of this level. Constraint optimization modelers like IMP ${ }^{5}$ procedural and analytical modeling approaches like Molecular Silverware ${ }^{6,7}$ and relaxation approaches like Brownian Dynamics modeling ${ }^{8,9}$ are currently used to build large-scale models. However, they are designed to function locally and do not model ultrastructural features like organelle membranes or fibrous molecules like actin. Qualitative manual protocols that use a more artistic approach have typically been required to produce mesoscale models. For example, when a large multidisciplinary collaboration determined the recipe for an average synaptic vesicle, the assembly of a structural model used to visualize the results of this effort took weeks of work using a combination of manual positioning with physics solvers to minimize collision. ${ }^{10,75}$

For the past twenty years, we have taken a semi-quantitative approach for the creation of illustrations of the cellular mesoscale, which integrate data across the nano and microscales. Recipes consisting of structures, concentrations and locations of molecules, macromolecules, and organelles taken from published reports are arranged into mesoscale compositions consistent with ultrastructural data from light and electron microscopy. ${ }^{\mathbf{1 1 , 1 2}}$ The illustrations are created manually with a $2.5 \mathrm{D}$ metaphor, typically presenting viruses or cells in crosssection, with simplified representations for the individual molecules. Fig. 1A shows a recent model of HIV-1 created with this method for educational outreach at the Protein Data Bank. ${ }^{13}$

The data used to specify this illustrated model served as the basis for the HIV-1 cellPACK recipe and 3D model. cellPACK computationally automates the modeling steps of the semi-quantitative artistic approach to extend the results from $2 \mathrm{D}$ paintings into 3D models that can be explored, animated, simulated, 
(A)

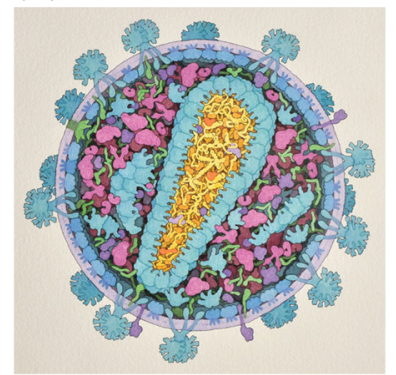

(B)

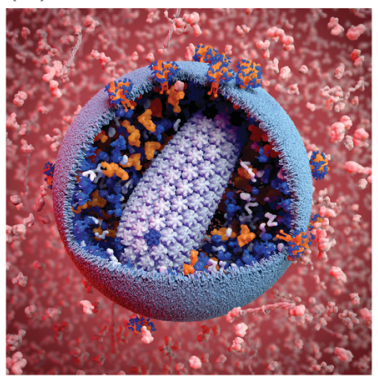

Fig. 1 Mesoscale Models of HIV-1. (A) A hand-drawn illustration of HIV-1 integrates data from structural biology, biophysics and microscopy. Data sources from this illustration were later used to create the digitized starting point recipe HIV-1_0.1.0. (B) A 3D model of HIV-1 enables exploration, analysis, animation and simulation, as well as easy variation, modification and updating. This model is based on the cellPACK recipe HIV-1_0.1.4 nested in the larger environment recipe of HIV_in_Blood_Plasma_0.1.2, with a custom lipid bilayer added by the artist. The image was created using the cellPACK viewer plugin by digital artist Andrew McWhae for the autoPACK Visualization Challenge 2013, an outreach project which challenged artists to "convey humanity's complex relationships with this virus, be they emotional, political, or intellectual... to excite general audiences with visuals that will help us spread interest in the search for a cure." (http://autopack.cgsociety.org)

analyzed, and easily edited and updated (Fig 1B shows a rendering of a 3D model created from the fourth iteration of the digitized recipe called HIV-1_0.1.4 (recipes use a 3 digit semantic versioning system to track them in a database where $H I V-1 \_0.1 .4$ stands for a recipe named "HIV-1" version 0.1.4).

\section{cellPACK can model the mesoscale at the pace of HIV research}

HIV structural biology is currently an area of intense research, with new breakthroughs reported each month. A major goal of cellPACK is to provide a nimble interface for integrating new structural results into the body of available data and evaluating their consequences on the integrative models. For instance, Fig. 4-6 describe two major iterative updates to the HIV-1 recipe, where HIV-1_0.1.6 in Fig. 6 includes structural updates to the envelope glycoprotein, capsid, and other HIV proteins. In addition, cellPACK is designed to allow exploration of ambiguity or imprecision in the various components of the recipe, creating a range of models consistent with the current state of knowledge. Fig. 3, for example, shows 3D structures of HIV that result from varying the parameters affecting surface protein distributions and quantities.

Modeling an organism involves two conceptual steps: gathering of data to create a recipe for the model, and use of this recipe to build a virtual model. The goal of this report is to describe the creation of a recipe and model for a mature HIV-1 particle, which will become part of a larger HIV-in-blood-plasma recipe, including details on all of the viral and host macromolecules. This document presents the references used to support the structure, location and interactions of molecules depicted in the rendered models. Several recent review articles provided an overview to the topic and sources for additional information. ${ }^{\mathbf{1 1 4 - 1 8}}$ 


\section{HIV-1 cellPACK recipes}

Data sources used to create the illustration in Fig. 1A were reused to create the cellPACK recipe HIV-1_0.1.0 as a driving biological project for cellPACK development. Digitization of the recipe involved building 3D representations of molecular components and other needed files, and associating parameters that drive localization, concentration, and interaction into the cellPACK recipe file format. The cellPACK recipe was corrected and updated periodically through four iterations, while developing the cellPACK software over a three-year period, to produce the recipe $H I V-1 \_0.1 .4$ visualized in Fig 1B. A major update to the recipe, created with the semi-quantitative approach, was published in $2012 ;^{19}$ this recipe was used to update $H I V$-1_0.1.4 to create $H I V-1 \_0.1 .5$, which is described in detail in the Results and in Fig. 4 and 5. Three major structural updates to the ingredients have since been published in 2013 and 2014, which were used, as noted in the Results, to update the recipe to HIV-1_0.1.6 (detailed in Fig. 6).

\section{cellPACK}

A full description of the cellPACK framework has been submitted for publication, meanwhile, details are available on the project website and online documentation at www.cellpack.org. In summary, cellPACK unites existing and novel packing algorithms to generate, visualize and analyze comprehensive 3D models of complex biological environments that integrate data from multiple experimental sources. cellPACK currently generates probabilistic 3D models of large sections of cells that can contain dozens to millions of molecules. It packs molecules and organelles into positions that satisfy constraints consistent with the input data across the full range of scales. cellPACK additionally provides tools to store, visualize, analyze and interact with the results to make mesoscale models and mesoscale modeling accessible to a variety of audiences.

As shown in Fig. 2, cellPACK framework defines a desired volume and stochastically packs it with objects, called ingredients, according to a recipe. Ingredients of arbitrary shape are placed into the allowable 3D space with zero or minimal overlap and with random distribution. Constraints and agent behaviors may be associated globally or with selected ingredients to add specific modes of interaction. A grid-based approach is used to generate models quickly while tracking and confirming global parameters. cellPACK's database consists of mesoscale recipes (e.g., cytoplasm, blood plasma, synaptic vesicles, HIV, etc.) and examples of models for each recipe.

To produce the models and visualizations shown in the figures, HIV-1 recipe ingredients (typically molecules) are stochastically packed into containers (polygonal meshes defining the envelope and capsid ultrastructures as described in the Results), up to densities provided for each ingredient, typically specific to a particular recipe. Each ingredient comes with associated properties, including a structural representation (such as a molecular surface ${ }^{20}$ or volume occupancy shell generated in this case with the molecular modeling software plugin $e P M V^{\mathbf{2 1}}$ ), and behaviors such as a particular collision-detection method or a list of binding partners that will modify the ingredient's global and local packing. For example, in $H I V-1 \_0.1 .5$ and $v 0.1 .6$, the MA proteins are restricted to pack on the inside 
A The containers of cellPACK recipe HIV-1_0.1.5 define surfaces and create volume boundaries
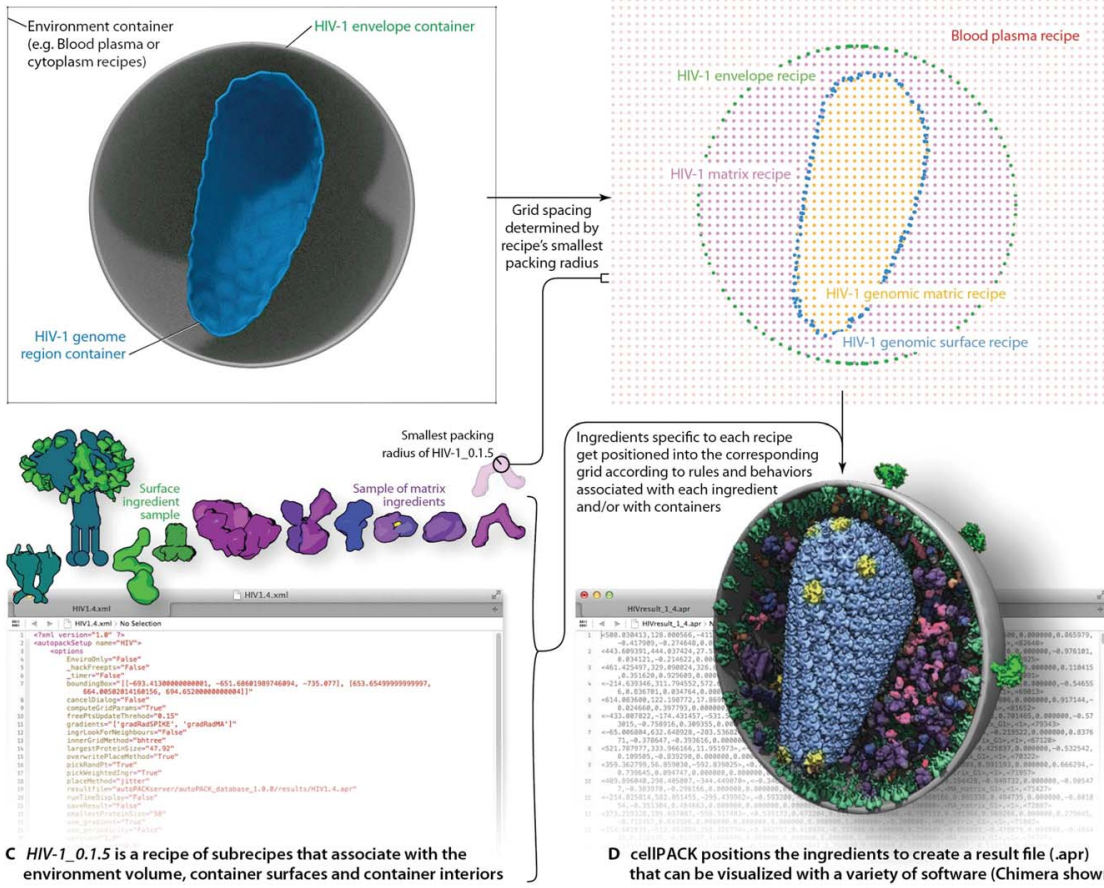

D cellPACK positions the ingredients to create a result file (.apr) that can be visualized with a variety of software (Chimera shown)

Fig. 2 cellPACK uses a grid-based method to track ingredients as they are efficiently packed into recipe-bounding containers. (A) Polyhedral mesh containers create boundaries to restrict the packing of HIV-1 envelope surface ingredients to the surface of the envelope mesh and HIV-1 matrix ingredients to the interior of the envelope and exterior of the capsid (HIV-1 genome region outer container). (B) An omniscient grid parameterizes the space to track ingredients as they pack and to weight regions for preferred packing based on global localization or distance measurements to ingredient interaction partners. The spacing of the grid is based on the radius of gyration of the smallest ingredient to pack. (C) The full recipe of ingredients is packed to (D) generate an .apr file with IDs and matrices for each ingredient packed. The apr file is used by a variety of visualization (e.g. molecular viewers such as Chimera shown in D) or animation software packages (e.g. Cinema 4D, Maya, Blender, 3D Studio Max, etc.) to rebuild the scene in an interactive viewport where objects can be turned on/off, replaced with more detailed data such as the original PDB file for an ingredient, or analyzed with a collection of cellPACK analysis tools or default analysis tools native to each viewer.

surface and have a higher probability of packing towards one end of the envelope bilayer as a first-draft global packing constraint that can recapitulate the emergent properties of molecular interactions that have been observed as ENV clustering in fluorescence microscopy. ${ }^{27}$ The polarization of ENV can be achieved by several methods currently under testing that include simplistic global constraints that force MA or ENV to pack preferentially towards some target location as demonstrated in Fig. 1B, as well as molecular scale interactions that vary association probabilities between MA-MA, MA-ENV, and ENV-ENV. Variations in the ingredient or recipe parameters can produce different results (Fig. 3). In the resultant model for a given recipe, each ingredient retains a connection to various other forms of data to enable deeper analysis, preparation for systems integration 


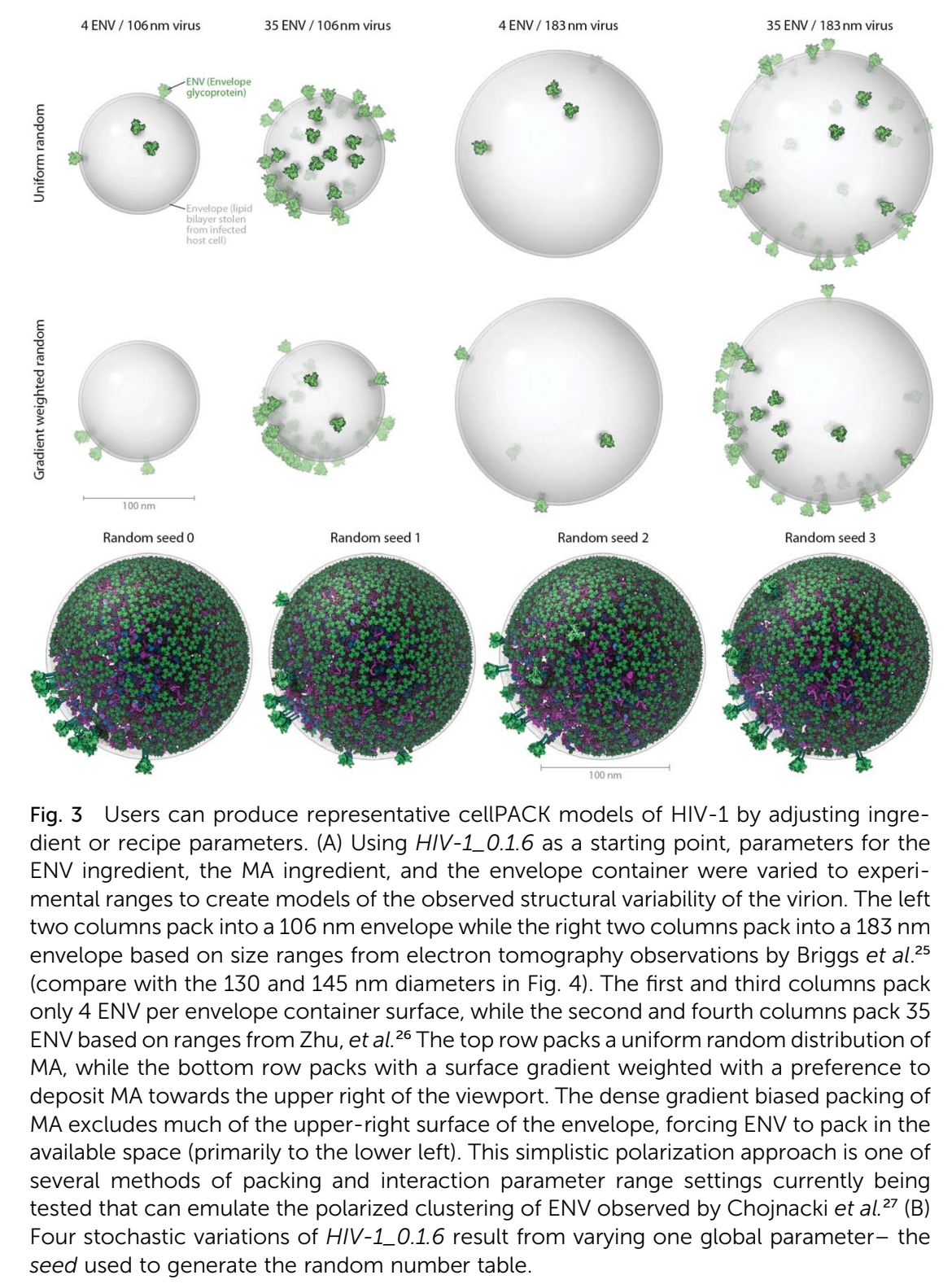

or large-scale simulations, or for modifications of the ingredient representations. The representations use coarse molecular surfaces by default, but can be replaced with an interactive molecular modeling session to modify the representation style and to access the atomic details from the structural files, for example, from Protein Data Bank (PDB) files or Electron Microscopy Data Bank (EMDB) files.

Unique cellPACK recipes, created for each version of HIV-1, or HIV_in_Blood_Plasma, served as input to the cellPACK software. Packing the recipes with any version of cellPACK (stable release 1.0 and newer) produces the resulting models presented in this paper as autoPACK Result (apr) files. An .apr file contains 
a list of ingredient IDs with positions and orientations as described in the online documentation (http://www.autopack.org/documentation/-apr-autopack-resultfile-anatomy). As specified in the legends, visualization software including the molecular viewers PMV ${ }^{22}$ and Chimera ${ }^{23}$ and a collection of cellPACK- $u P y^{24}$ pluginenabled 3D animation packages were used to load and visualize the model defined in the HIV-1_0.1.5 and v0.1.6 apr files. The visualization software all use the same method of downloading the default geometric representation for each unique recipe ingredient followed by generating efficient copies of that representation on the computer's graphics card to enable real-time viewing and fast rendering to create static images, animated sequences, or online viewers.

\section{Community consensus on theoretical mesoscale models}

cellPACK provides scripting and GUI interfaces to access viewing, analysis, and modeling modules. A database, scheduled to go online in the Summer of 2014, will soon enable invited users to critique and modify recipes and ingredients while maintaining version histories directly through the project website. Having been through numerous internal edits, upon publication, the $H I V-1 \_0.1 .6$ recipe will become the first full-release (version 1.0) of HIV in the semantic versioning system (laterally named as HIV-1_1.0.0) and the first official model in the database to be opened for community feedback. Through web browser interfaces and leveraging social-networking technologies, the project website will ask the scientific community to analyze, critique and edit the models in an effort to produce confidencevoted community consensus models and to iteratively improve the recipes.

\section{Results}

\section{cellPACK models track progress in HIV structural research}

The modular and hierarchical architecture of cellPACK is designed to streamline the incorporation of the most current experimental results into representative models. The modules of cellPACK correspond to different experimental techniques with widely different data types. Ultrastructural data from electron microscopy is captured in the containers. Biophysical data on concentrations and localization are captured in the recipes. Structural data is captured in the models for each ingredient. This modularization allows new results to be efficiently added to an existing recipe.

We have included several examples of how this modularity can provide tools for exploring the structural biology of a biological system. Fig. 4 shows the models created with our two most recent recipes for HIV-1, HIV-1_0.1.5 and HIV-1_0.1.6. Version 0.1.6 incorporates newly published results for the envelope glycoprotein (ENV), VIF, and capsid that were not available when the previous recipe was created. This figure visualizes the full HIV particles, with sections removed to reveal their internal components. Fig. 5 and 6 chart individual depictions of the molecular ingredient representations where Fig. 5 details the specifics of the vo.1.5 recipe and Fig. 6 highlights the changes between the two versions.

Fig. 3A shows how cellPACK can be used to explore experimental uncertainties or ranges of parameters in experimental data. HIV is a pleomorphic organism, with a range of sizes and numbers of component molecules. cellPACK is able to create models that represent extrema in these ranges or that represent virions 
A Rendered 3D model that resulted from the packing of cellPACK recipe HIV-1_0.1.5

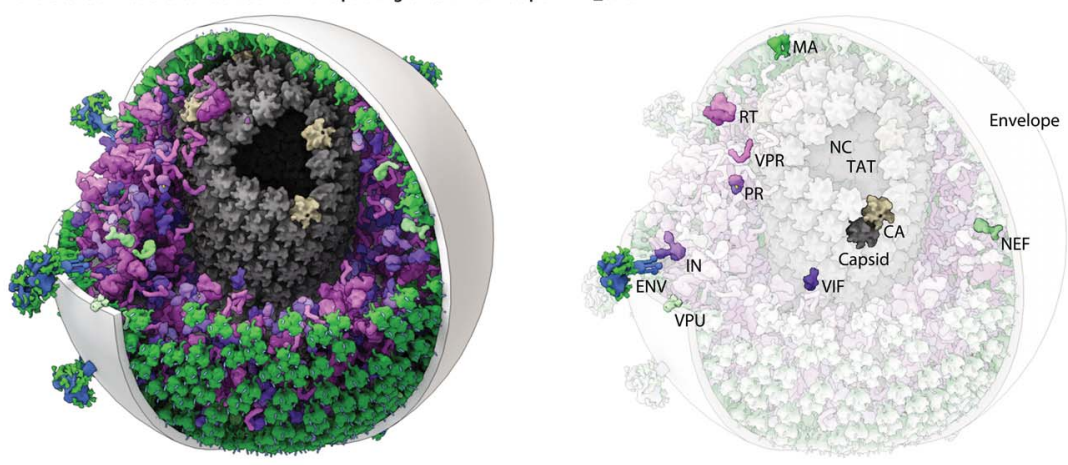

B Rendered 3D model that resulted from the packing of cellPACK recipe HIV-1_0.1.6
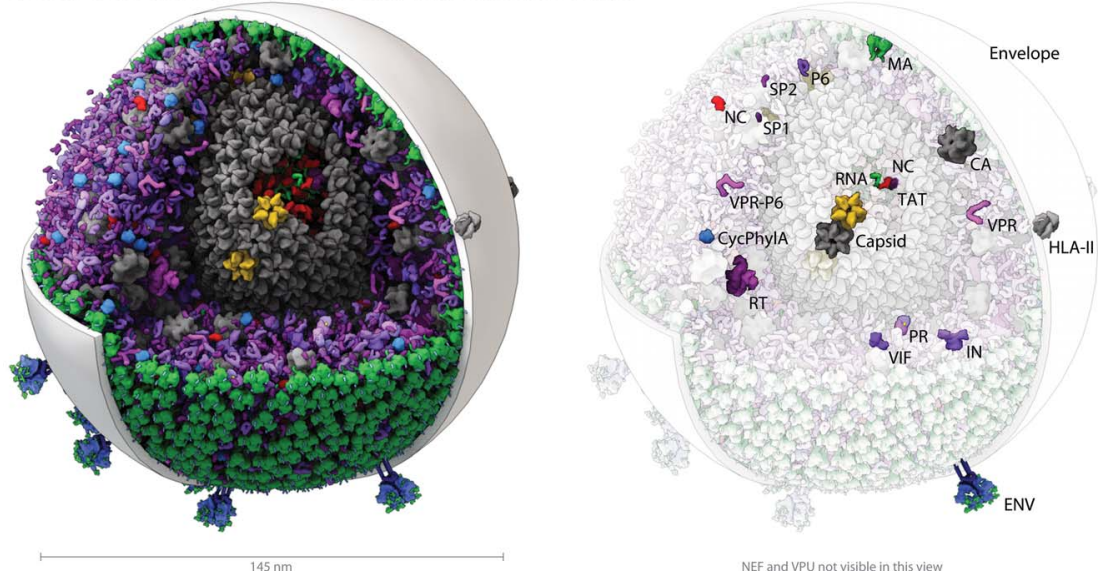

Fig. 4 A visual comparison of HIV-1_0.1.5 and HIV-1_0.1.6 reveals consistent, but stochastic packing where ingredients primarily change in their intramolecular structure and quantity rather than in location or localization. (A) The HIV-1_0.1.5 recipe provides a substantial update to the HIV-1_0.1.4 recipe shown in Fig. 1B with numerous ingredient corrections and additions. The model, visualized in a uPy-enabled 3D animation software package shows six different recipes (each a different color coding) unified into a single model. (B) Three major structural updates as well as more subtle quantification and envelope dimension changes were added to update HIV-1_0.1.5 to HIV-1_0.1.6.

with average properties. Finally, Fig. 3B shows that cellPACK is able to create multiple representative models based on a given recipe, stochastically exploring the range of possible structures that are consistent with the given description. Packing times vary widely across recipes depending on the methods specified, the size and detail of the ingredients, the packing volume size, the total ingredient density, the complexity of the interactions, etc. Each of the four models in Fig. 3B pack the default v0.1.6 recipe using a robust collision algorithm (the industry standard Bullet Physics Engine, bulletphysics.org) that allows zero ingredient surface overlaps. The models in Fig. 3B, which vary only in the random seed, each took $<267$ seconds to pack on a standard consumer 2012 laptop computer. 


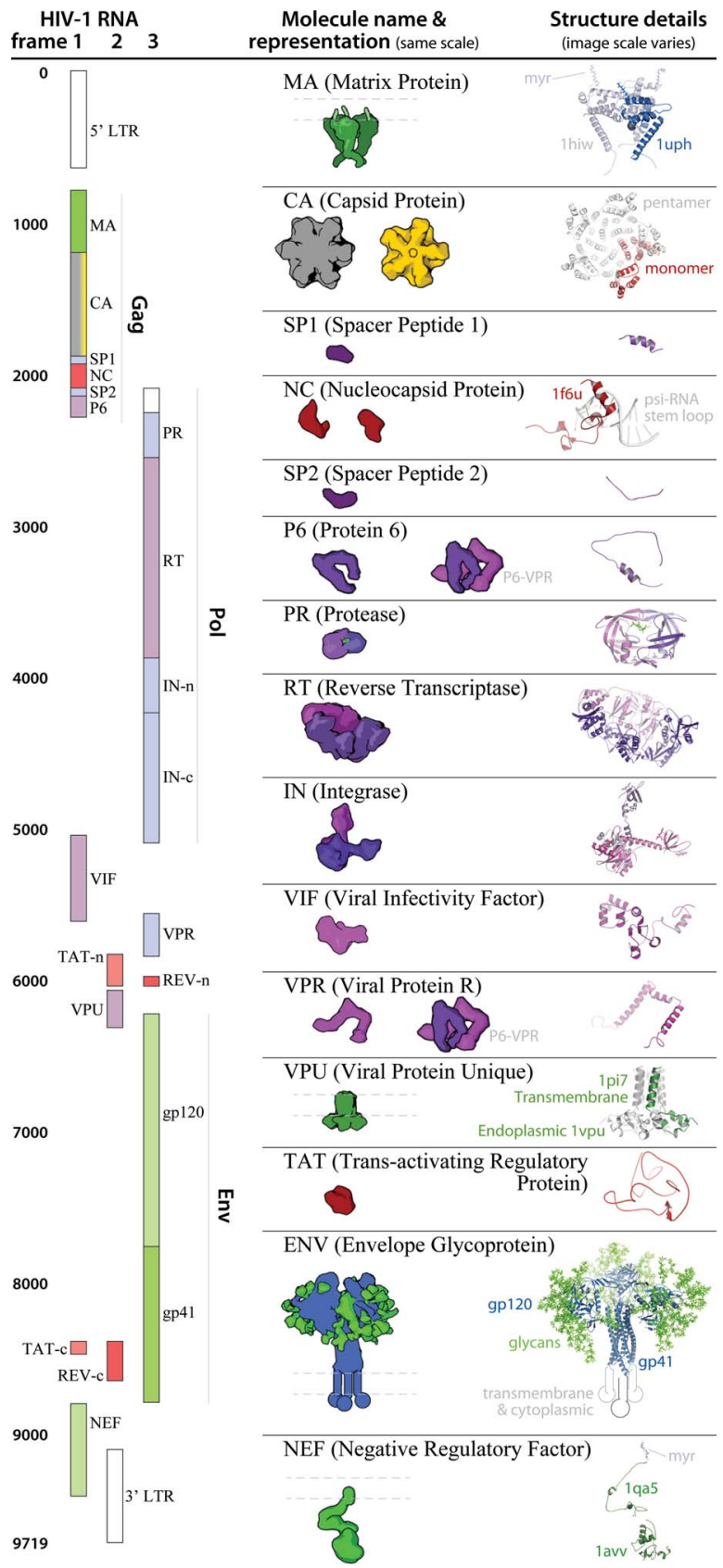

Fig. 5 HIV-1_0.1.5 ingredient representations with structural summaries. The HIV genome landmark map on the left side (redrawn from www.hiv.lanl.gov/content/ sequence/HIV/MAP/landmark.html) relates to the default coarse molecular surface representations and structural details of the HIV gene products in the center column. Many of the ingredients are updated in the newer iteration of this recipe, HIV-1_0.1.6 as detailed in Fig. 6. 


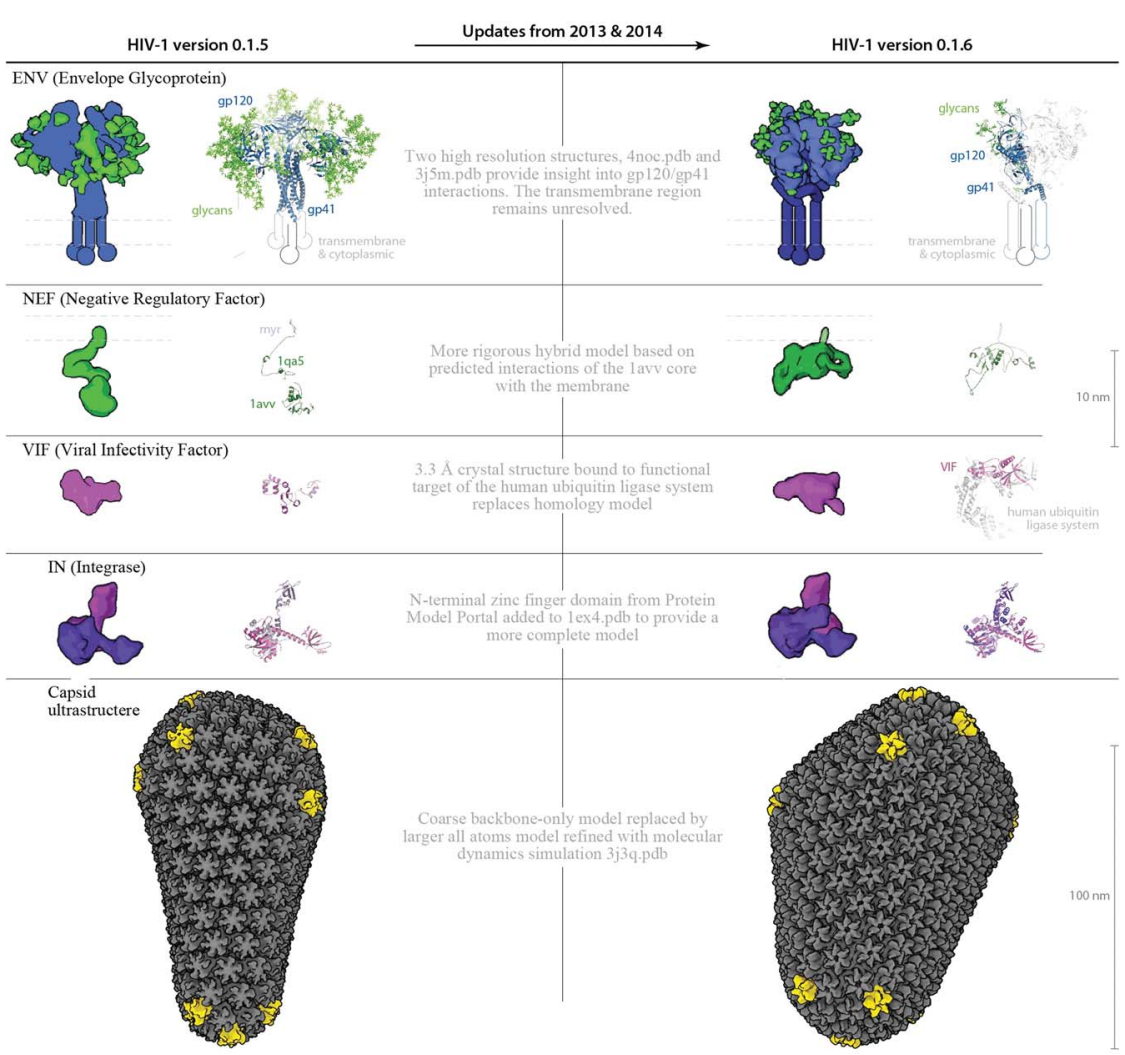

Fig. 6 Structure and parameter changes between HIV-1_0.1.5 and HIV-1_0.1.6. Two ingredients ENV and VIF, and the ultrastructural capsid have been updated with new structures published in the past 9 months. The new structures provide important targets for vaccine and drug design, and because there may be steric hindrances imposed by their interactions or localizations, its important to visualize the ingredients in the context of the whole virus. Improved homology models for NEF and IN provide more complete representations for these two ingredients. The left column shows "older" structures and/or parameters from HIV-1_0.1.5 that were changed to iterate the recipe to the "upgraded" HIV-1_0.1.6.

The remaining portion of the results will present the sources that were used for creation of the HIV-1_0.1.5 and v0.1.6 recipes, revealing some of the challenges for specifying this type of recipe and identifying several gaps in knowledge where approximations were required. This portion simultaneously includes a brief description of the known functions of each ingredient to provide a comprehensive resource that will be folded into the developing database.

\section{Recipe biology, structure, and parameter details}

\section{Ultrastructure: Envelope container and matrix}

HIV envelopes are lipid bilayers, captured from the host cell during release, that encapsulate the virus, display the viral ENV proteins, and shield most of the viral machinery from the host immune system. Based on electron micrographs, HIV 
Table 1 Published viral envelope diameters

$$
\begin{aligned}
& 133 \pm 17 \mathrm{~nm}(n=118) \text { (authentic immature virions) }{ }^{28} \\
& 145 \pm 25 \mathrm{~nm}(n=255) \text { (mature virions) }{ }^{29} \\
& 139 \pm 16 \mathrm{~nm}(n=197) \text { (immature virions) }^{29} \\
& 135.0 \pm 17.4,116.9 \pm 8.5(n=10-15 \text {, mature virions, two cell lines })^{30} \\
& 125 \pm 14 \mathrm{~nm} \text { (106-183) (mature virions) }{ }^{25} \\
& 119 \pm 207 \mathrm{~nm}(95 \% \text { of mature virions })^{31} \\
& 110 \pm 8 \mathrm{~nm} \text { (mature virions) }{ }^{26} \\
& 126 \pm 10 \mathrm{~nm}(n=30 \text { tomogram })^{32} \\
& 130 \pm 34 \mathrm{~nm}(n=150 \text { cryoEM })^{32} \\
& 128 \pm 32 \mathrm{~nm}(n=84 \text { STEM })^{32} \\
& \text { 100-150 nm (review article) }{ }^{14}
\end{aligned}
$$

particle envelopes are primarily spherical, but pleomorphic with occasional subtle lumps in the flexible lipid bilayer surface, and the envelope diameters range from $100 \mathrm{~nm}$ to $207 \mathrm{~nm}$ (Table 1). Early cellPACK recipes for HIV used distorted spheres (see Fig. 1B) to capture the observed variations in shape, but to simplify analysis (such as the angular distribution variance of ENV) and to describe more average HIV particles, the recipes for $H I V-1 \_0.1 .5$ and v0.1.6 use perfect spheres as containers for the envelope packing surfaces. $H I V-1 \_0.1 .5$ envelope has a diameter of $130 \mathrm{~nm}$, measuring to the center of the lipid bilayer. $H I V-1 \_0.1 .6$ has a diameter of $145 \mathrm{~nm}$. The bilayer, modeled after MD simulated patches, ranges from $2-5 \mathrm{~nm}$ in thickness across the hydrophobic region (http:// nbcr.ucsd.edu/data/sw/hosted/lipidwrapper). Most of the figures show the bilayer as a $3 \mathrm{~nm}$ thick spherical shell for visual clarity.

The envelope bilayer in both recipes is populated by both viral and host molecules. The region inside the envelope but excluded by the capsid is termed the matrix. In both of the models, the matrix is populated with a collection of soluble viral and host molecules.

\section{Ultrastructure: Capsid container and genomic region}

A distinctive cone-shaped capsid protects the HIV genome (2 strands of RNA visible in Fig. 4B) and delivers the genome to new host cells upon infection. The entire capsid is built from repeating units of a single capsid protein. The large $\mathrm{N}$-terminal domain of a capsid protein binds to other copies of capsid proteins to form stable hexameric rings ${ }^{33}$ and occasional pentameric rings. ${ }^{34}$ The C-terminal domain links rings to neighboring rings to form the conic HIV capsid superstructure. HIV capsids have been studied by electron microscopy (EMDB entry 5639) and tomography ${ }^{25}$ and have been reconstructed as atomic models using hybrid methods in two major publications. HIV-1_0.1.5 uses a smaller coarse, backbone-only model provided by Pornillos and Yeager ${ }^{34}$ that stands $\sim 115 \mathrm{~nm}$ tall with 1056 monomers (166 hexamers and 12 pentamers, coordinates provided by the authors). HIV-1_0.1.6 uses the larger of two all-atoms models that were refined with molecular dynamics, which stands $\sim 118 \mathrm{~nm}$ tall, but is wider and contains 1356 capsid monomers (216 hexamers and 12 pentamers) comprised of 2440800 atoms $^{3}$ (PDB entry 3j3q).

The interior of the capsid holds the genome and associated proteins. In our models, we have termed this space the genomic region. Each capsid monomer has a unique conformation. To provide a coarser level of detail useful for interactive 
visualization, the $H I V-1 \_0.1 .6$ model uses a single representative monomer (chain A of $3 \mathrm{j} 3 \mathrm{q}$ ) aligned to each of the 1356 positions. The full detail PDB model with the unique conformers can be overlaid since the coarse model uses the same coordinate system.

\section{Recipes: Molecules and interactions restricted to container boundaries}

As new HIV particles bud from the infected host cell, the majority of the viral proteins are found in Gag (p55) and GagPol (p160) polyproteins (a note on nomenclature: the "p" and "gap" names are based on experimental molecular weights, and have largely been replaced by the more descriptive molecular names). Upon maturation, HIV protease (PR) cleaves each Gag and GagPol into 6 and 9 functional proteins respectively. Reports on Gag and GagPol quantification provide the most reliable abundances for proteins derived from Gag or GagPol in both mature and immature virus models. A wide range of values for the number and distribution of Gag and GagPol have been reported. Most current review articles have settled on a value of $2500 \mathrm{Gag}$ polyproteins and a ratio of $20 \mathrm{Gag}: 1$ GagPol, with some primary sources listed in Table 2. HIV-1_0.1.5 assumes 1710 Gag with $90 \mathrm{GagPol}$ for a total of 1800 polyproteins. HIV-1_0.1.6 uses $2375 \mathrm{Gag}$ and $125 \mathrm{GagPol}$ for a total of 2500 polyproteins.

\section{Recipes from $\mathbf{N}$ to $\mathrm{C}$ : Molecules and interactions from Gag proteins}

The Gag region of the HIV genome includes the proteins MA, CA, SP1, NC, SP2, and P6. HIV-1_0.1.5 packs 1800 copies of each Gag region protein. HIV-1_0.1.6 packs 2500 copies of each Gag region protein.

MA [envelope inner bilayer surface] - p17. In immature HIV-1 virions, the matrix protein (MA) localizes Gag and GagPol polyproteins to the host cell membrane, binding to the lipid bilayer through a covalently attached myristoyl chain. Mature MA forms trimers and remains associated with the membrane, but its major role in assembly appears to be the membrane attachment rather than the

Table 2 Gag and GagPol quantification and ratio reports Gag : GagPol ratios

$20 \mathrm{Gag}: 1 \mathrm{GagPol}$ (quantified on gels ${ }^{35}$ )

$89 \% \mathrm{Gag}: 11 \% \mathrm{GagPol}$ (shorter construct, quantified on gels) ${ }^{36}$

$2000 \mathrm{Gag}: 200 \mathrm{GagPol}^{28}$

\section{Number of Gag}

$2000 \mathrm{Gag}^{37}$

1008,1512 (calculated from experimental Gag : env ratio and counting of ENV on tomograms $)^{30}$

$4900 \mathrm{Gag}$ (8 $\mathrm{nm}$ lattice at $60 \mathrm{~nm}$ radius in $145 \mathrm{~nm}$ particle, no gaps $)^{31}$

$2400 \pm 700$ (70\% coverage, tomography $)^{32}$

$2400(61 \% \text { coverage, STEM })^{32}$

$2500 \mathrm{Gag}$ (review article) ${ }^{14}$

\section{Distribution of Gag}

$40 \%$ coverage $(28 \%-60 \%)$ (estimated visually from tomograms) ${ }^{38}$ $60 \% \pm 18 \%$ coverage $(n=64 \text { cryoTEM, sucrose purified virion })^{39}$ $82 \% \pm 16 \%$ coverage $(n=64 \text { cryoTEM, OptiPrep purified virion })^{39}$ $2 / 3$ coverage, continuous, with one gap (reviewed) ${ }^{15}$ 
ability to oligomerize. ${ }^{14} H I V-1 \_0.1 .5$ and v0.1.6 use a hybrid model for MA assembled using two PDB files. An NMR monomer with N-terminal myristoyl (PDB entry 1uph) and more complete sequence was superimposed onto the truncated crystallographic structure of the MA trimer (PDB entry 1hiw). The covalently attached myristoyl group, which is bound in a surface pocket in the soluble NMR structure, was rotated about the myr-amino acid bond to allow insertion into the membrane. MA also interacts with the membrane via interaction with the cellular lipid PI(4,5)P2, ${ }^{40}$ which is not included in the current models. MA is distributed on the inner leaflet of the envelope bilayer with a weighted envelope gradient that polarizes the distribution stochastically towards one side of the envelope in both $H I V-1$ - 0.1.5 and v0.1.6. A packing module in development packs MA trimers into hexameric $\mathrm{c}^{41,42}$ and random clusters to recapitulate more recent observations $\mathrm{s}^{43}$ and to enable denser MA packing. $H I V-1 \_0.1 .5$ packs 600 MA trimers (from 1800 precursor monomers). $H I V-1 \_0.1 .6$ packs 833 MA trimers.

CA [capsid, matrix, genomic region] - p24. Capsid protein (CA) forms the coneshaped capsid that protects and delivers the HIV genome. HIV-1_0.1.5 uses structural models pulled from crystallographic structures of the hexamer and pentamer, with backbone-only structural files provided by Mark Yeager (from Pornillos et al. ${ }^{34}$ ). $H I V-1 \_0.1 .6$ uses subunits A, B, C, D, E, and F pulled from PDB entry 1vu4 (partial structure of the entire capsid $3 \mathrm{j} 3 \mathrm{q}$ described under the Ultrastructures section above). HIV-1_0.1.5 has a conic capsid with 1056 monomers leaving $1800-1056=$ 744 CA monomers in solution in the matrix, and HIV-1_0.1.6 has a conic capsid with 1356 monomers leaving $2500-1356=1144$ CA monomers in solution in the matrix. The cellPACK recipes select from a library of hexamers, and various clusters of associated hexamers (partial capsids) that are packed into the matrix or genomic region until the respective limits are reached. A packing module under development, but not used here, can pack weighted assemblies of partial capsids on the fly directly from the hexameric subunits to enable the partial capsids to interact with other structures in the matrix and genomic region.

SP1 [matrix] - p2. Spacer Peptide (SP1, also known as P2) acts as a spacer between the CA and NC subunits in Gag and GagPol precursors. Although its role is poorly understood, SP1 is essential for viral assembly and infectivity. ${ }^{44,45} \mathrm{HIV}$ 1_0.1.5 and v0.1.6 model SP1 as an unstructured polypeptide 14 amino acids long. Structural studies are incomplete but suggest that SP1 may equilibrate ${ }^{46}$ between a random coil and an $\alpha$-helix. ${ }^{47}$ In immature viruses, SP1 forms a 6-helix bundle in the Gag lattice that includes the last 12 CA residues. ${ }^{38}$

NC [genomic region bound to RNA] - p7. Nucleocapsid protein (NC) interacts specifically with packaging signals (see below) and nonspecifically with viral and host RNA. 5-7 RNA nucleotides per Gag is sufficient for assembly of virus-like particles of $\mathrm{Gag}^{48}$ and a 5 nucleotide minimum binding site was identified by SPR on small oligonucleotides. ${ }^{49} \mathrm{NC}$ for both $H I V-1 \_0.1 .5$ and v0.1.6 is assembled from PDB entries 1a1t (from a complex with a psi loop) and 1f6u (from a complex with an SD stem loop). A total of $1700 \mathrm{NC}$ pack into the genomic region of $H I V-1 \_0.1 .5$, with 100 in the matrix and a total of $2300 \mathrm{NC}$ pack into HIV-0.1.6 with 200 in the matrix.

SP2 [matrix] - p1. Spacer Peptide (SP2, also known as P1) separates NC from P6 and seems to be essential for viral infectivity. Deleting SP2 leads to defective capsid construction that may be related to kinetic effects in the C-terminal region of Gag. ${ }^{50} H I V-1 \_0.1 .5$ and v0.1.6 use the same representation of SP2 modeled as an unstructured 17 amino acid polypeptide. 
P6 [matrix] - p6. P6 docks several viral and host-cell proteins and may associate with the envelope membrane. ${ }^{51}$ Many studies suggest that P6 remains largely unstructured in the mature virion, and interacts with VPR. ${ }^{37} H I V-1 \_0.1 .5$ and v0.1.6 split the number of P6 into two representations with different densities: one homology model (from the P6 FASTA sequence templated to PDB entry 2c55 via Swiss-Model ${ }^{52}$ ) with a partially helical and mostly unstructured chain of 51 amino acids (1500 copies), and a second representation with this homology model proximal to VPR (200 copies).

\section{Recipes: Molecules and interactions from the pol region of GagPol}

Roughly $5-11 \%$ of the Gag polyproteins are extended by a translational frameshift to create the GagPol polyproteins ${ }^{36}$ which contain three additional functional subunits (PR, RT, and IN) that are processed by HIV-1 protease to create the mature enzymes. HIV-1_0.1.5 packs 90 copies of each pol region protein. $H I V$ 1_0.1.6 packs 125 copies of each pol region protein.

PR [matrix]. Protease (PR) is a dimer of identical subunits, and is involved in the processing of Gag and GagPol during the process of maturation. HIV-1_0.1.5 and v0.1.6 use a crystallographic structure of the dimer from PDB entry 7hvp.

RT [matrix and genomic region] - p51. Reverse transcriptase creates a DNA copy of the HIV-1 RNA genome, which is then inserted into the host genome and used to create progeny virions. HIV-1 reverse transcriptase is a heterodimer of the RT protein and a truncated version of the protein. HIV-1_0.1.5 and v0.1.6 use a crystallographic structure of the heterodimer from the PDB entry 3hvt.pdb. Both recipes locate 25 of the $125 \mathrm{RT}$ in the genomic region.

IN [matrix and genomic region] - p31. Integrase enzyme (IN) splices the DNA copy of the viral genome into host cell chromosomes. Full-length integrase, which functions as a homotetramer, has yet to be crystallized. HIV-1_0.1.5 uses a partial model of IN based on the core structure in PDB entry 1ex4, which is missing the 450-amino-acid zinc finger domain from the $\mathrm{N}$ terminus. HIV-1_0.1.6 uses a composite model with the $\mathrm{N}$-terminal zinc-finger region from Protein Model Portal entry a510eadb0df840e958233f484781dde7_7 superimposed onto the C-terminal and core structure of PDB entry 1ex4. Both recipes locate 25 of the $125 \mathrm{IN}$ in the genomic region.

\section{Recipes: Molecules and interactions from the envelope region}

Envelope glycoprotein ENV [envelope bilayer surface] - gp160. Envelope glycoprotein (ENV) forms trimers that protrude outward from the envelope surface. ENV contains the cellular receptor binding sites and membrane fusion machinery that power entry of the viral contents into the cytoplasm of a new host cell upon infection..$^{53}$ After binding to CD4 receptors on the surface of a cell, ENV snaps into a different conformation that pulls the viral envelope membrane close to the plasma membrane of the cell until the two bilayers fuse.

Three identical subunits trimerize to form each ENV spike and each trimer has $\sim 81$ glycosylation sites that cover ENV with a shielding layer of carbohydrates that comprises up to $50 \%$ of the mass of each spike. $H I V-1 \_0.1 .5$ uses an older chimeric model templated from $2 \times 7 \mathrm{r}, 1 \mathrm{~g} 9 \mathrm{~m}, 3 \mathrm{dnn}$ and glycosylated with glycprot server (http://www.glycosciences.de/modeling/glyprot/php/main.php). HIV-1_0.1.6 uses a mix of two structures of the ectodomain, a crystal structure (PDB entry $4 \mathrm{nco}$ ), and a 
single-particle electron microscope structure (PDB structure $3 \mathrm{j} 5 \mathrm{~m}$ ). The new structures contain part of the gp41 stalk in the prefusion state, which provides insights into trimer assembly and the quaternary interaction between gp120 and gp41. The structures also contain bound broadly neutralizing antibodies as a focus for vaccine design. Some of the representations in the model include these antibody (Fab) fragments. The transmembrane region has not been resolved experimentally and is represented by geometric shapes of approximate size and shape based on molecular mass and positioned in close proximity to one another, based on electron tomography. ${ }^{54}$

The number and structure of ENV projecting from the virion surface has been difficult to quantify (see Table 3 for reported variations). Current reviews state that there are about 10 trimers on the surface. ${ }^{15} H I V-1 \_0.1 .5$ packs 21 ENV while $H I V$ 1_0.1.6 reduces the number to 12 for the $145 \mathrm{~nm}$ virus. Fig. 3 shows models that result from varying this number across the published extremes (from 4 to 35).

The arrangement of ENV on the surface is a topic of current research. Fluorescence studies found a single cluster in mature virions, but not in immature virions. ${ }^{27} H I V-1 \_0.1 .5$ and v0.1.6 pack MA along a preferential gradient that polarizes the distribution of ENV stochastically toward one side by stochastically excluding much of the surface otherwise available to ENV.

Interactions with molecules inside the virion also remain controversial. One study found that cellular protein TIP47 bridges MA and ENV and is required for packaging, ${ }^{57}$ but a later paper found that MA and TIP47 interact, but is not needed for ENV incorporation. ${ }^{58}$ Based on a current report, the "mechanism of incorporation still remains unclear": they present genetic evidence for ENV and MA interaction, but also state "it would seem plausible that passive incorporation could be sufficient to account for the $\sim 10$ copies",59 therefore the recipes and resulting models do not yet include any specific interactions.

\section{Recipes: Molecules and interactions of HIV-1 accessory proteins}

VPR [matrix]. Viral Protein $R$ (VPR) is an accessory protein with multiple functions critical for effective infection of CD4 cells and macrophages. VPR transports the HIV-1 pre-integration complex into the nucleus, arrests the cell cycle, and initiates transcription of HIV-1 LTR. ${ }^{60}$ The 96 amino acid VPR representation for HIV-1_0.1.5 and v0.1.6 is based on PDB entry 1esx. VPR is incorporated into virions through interaction with Gag at a concentration of 1 VPR : 7 Gag' ${ }^{61}$ equaling 257 VPR in HIV-1_0.1.5 and 357 VPR in HIV-1_0.1.6. 200 of the VPR in the matrix, remain bound to $\mathrm{P} 6 .^{37}$

Table 3 Published quantities of ENV/virus

70-80 “projections"55

7-14 trimers (measured Gag : ENV 60 : 1, assume 1200-2500 Gag) ${ }^{56}$

8,10 (range of $3-12,5-17$, counted on tomogram from two cell lines) ${ }^{30}$

14 spikes (range $4-35, n=40$, in several clusters, counted on tomogram) ${ }^{26}$

Table 4 NEF quantification in HIV

$<70$ full length + cleaved per virion (quantified on gels ${ }^{64}$ )

$1 \mathrm{NEF}$ : 10-20 pol, $1 \mathrm{NEF}$ : 333-200 Gag, 5-10 NEF per viron (assuming $2500 \mathrm{Gag})^{35}$ 
NEF [envelope bilayer inner surface, matrix]. Negative Regulatory Factor (NEF) modifies host cell structures and functions to favor replication and pathogenicity. $H I V$-1_0.1.5 uses a hybrid model that positions the myristoyl group from the $\mathrm{N}$-terminus of PDB entry 1qa5 in the membrane and positions the C-terminal core of PDB entry 1avv near the distal end. HIV-1_0.1.6 uses a homology model interacting more closely with the bilayer, ${ }^{62}$ generated with Modeller ${ }^{63}$ based on the crystallographic structure of full-length NEF in PDB entry 3tb8, which includes only backbone atoms. The myristoyl group was taken from PDB entry 1qa5 and manually aligned into the bilayer using PyMol. Several studies have addressed the amount of NEF incorporated into virions (see Table 4):

NEF appears to be bound to the membrane in the immature virion, but the "majority" ${ }^{64}$ or $50-70 \%{ }^{35}$ is cleaved to soluble form in mature virion, as quantified on gels. HIV-1_0.1.5 packs 35 envelope bound NEF and 30 soluble NEF. $H I V-1 \_0.1 .6$ reduces this to 5 envelope bound NEF and 5 soluble.

VPU [envelope bilayer]. Viral Protein Unique (VPU) promotes CD4 degradation and release of the immature virus from the host cell plasma membrane. VPU forms a pentameric channel through the membrane. HIV-1_0.1.5 and v0.1.6 use a chimeric model assembled from the transmembrane region of PDB entry 1pi7 and the cytoplasmic region in PDB entry 1vpu. VPU is found primarily in the endoplasmic reticulum of the infected cell where it performs its job and little to none may escape with the immature virion, so both HIV-1_0.1.5 and v0.1.6 include only 20 copies (4 pentamers).

TAT/TAR [genomic region]. Trans-activating Regulatory Protein (TAT) increases viral replication efficiency by mediating the phosphorylation of cellular factors that increase the transcription of all HIV genes. TAT binds to the trans-activating response element (TAR) on the $5^{\prime}$ end of HIV transcripts and alters the transcription machinery to increase production of RNA. The TAT structure is taken from model 1 of the 86 amino acid NMR structure in PDB entry $1 \mathrm{jfw}$. Both recipes pack TAT at a similar density, with 45 TAT in HIV-1_0.1.5 and 54 TAT in the larger capsid of HIV-1_0.1.6.

VIF [matrix]. Viral Infectivity Factor (VIF) neutralizes host defenses by targeting the host enzyme APOBEC for ubiquitination and subsequent proteasomal degradation. HIV-1_0.1.5 fixes an incorrect structure from $H I V-1 \_0.1 .4$, which had been surfaced from the full model of PDB entry $3 \mathrm{dcg}$, which includes only a small fraction of VIF and large sections of a human elongation factor protein. $H I V$ 1_0.1.5 uses a homology model from ModBase model ID 369ab672da66deecac38810f962b4e53, templated from PDB entry 1f0xA that covers 1-174 out of 192 amino acids in VIF. HIV-1_0.1.6 improves further on the VIF model with a larger fragment of VIF (aa 1-176) from a crystallographic structure in PDB entry 4n9f, ${ }^{65}$ where it was crystallized bound to the large human ubiquitin ligase system (its functional target). The literature reports 7-100 VIF per virion ${ }^{37}$ and both $H I V-1 \_0.1 .5$ and v0.1.6 pack at a density of $100 \mathrm{VIF} / 145 \mathrm{~nm}$ virion.

Viral RNA [genome encased in the capsid]. The genome of HIV has 9749 nucleotides ${ }^{66}$ detailed on the left side of Fig. 5. The genome has several regulatory regions in addition to the coding regions, including the Psi region that directs selective packaging and dimerization and the major packaging signal with SL1, which contains the dimerization initiation site (DIS), and SL3. ${ }^{67}$ A prediction of secondary structure of entire genome has also been reported. ${ }^{68}$ HIV-1.6 includes a very coarse structure modeled by Goddard 
Table 5 A few specific interactions have been reported

Cyclophilin A $1: 10 \mathrm{Gag},{ }^{70}$ shown in the illustration based on the PDB entry $1 \mathrm{ak} 4$

(cyclophilin A and N-terminal domain of CA)

Hsp70 $1: 20 \mathrm{Gag},{ }^{69}$ bound to $\mathrm{Gag}$

INI1 $1: 2$ integrase, bound to integrase ${ }^{69}$

Ubiquitin $1: 10 \mathrm{Gag}^{69}$

Monoubiquinated proteins 1: $50 \mathrm{Gag}^{69}$

Lys tRNA synthetase, bound to Gag

TIP47 bound to $\mathrm{MA}^{57,58}$

ABCE1 bound to $\mathrm{NC}^{71}$

Staufen1 (ds RNA binding protein) bound to $\mathrm{NC}^{72}$

Annexin2 bound Gag (in macrophages) ${ }^{73}$

[http://tinyurl.com/ChimeraRNA] incorporating only the predicted secondary structure. This RNA model is included for size and to give an idea of the complexity of packing, but does not specifically interact with the capsid and is not a plausible packing usable for research purposes. Modeling of RNA is an active area of development for cellPACK.

Cellular RNA. Cellular RNA is incorporated into the virion through interaction with NC. ${ }^{16}$ For instance, 7SL (RNA from signal recognition particle) is found in a ratio of $7: 1$ with the HIV genome. HIV-1_0.1.5 and v0.1.6 do not include cellular RNA.

Cellular proteins. Many cellular proteins are incorporated into the virion (see Table 5), including ICAM1, HLA-II, and tetraspanins in the membrane, and soluble proteins such as thioltransferase, Hsp70, actin and actin-binding proteins, ubiquitin and lys tRNA synthetase. ${ }^{69}$

The HIV-1_0.1.6 recipe includes these examples, but is not yet comprehensive. We plan a detailed literature search and modeling effort in the next iteration to include the host proteins into the recipe and model. This is an area of intensive research, as the community is uncovering the roles of host proteins in the viral lifecycle.

\section{Conclusions}

cellPACK is designed to form a bridge between the approaches of reductionist biology and systems biology. As computational and experimental techniques probe into the mesoscale from smaller and larger scale levels, frameworks like cellPACK, that will unify and contextualize the diverse data, are needed to serve as platforms for consistent communication to facilitate interdisciplinary research. cellPACK models can serve as hypothesis-generating tools, as starting points for mesoscale simulation techniques like Brownian Dynamics, ${ }^{9}$ and have already been applied extensively for education and outreach.

Modeling a virus is a relatively simple task compared to modeling an entire eukaryotic cell in molecular detail. A complex virus like HIV serves as an ideal short-term goal, which now provides a much needed framework for the HIV research community to assemble their data and to better understand lentivirus mechanics. HIV simultaneously serves as a stepping-stone to modeling organisms of increasing complexity. The up-to-date release of HIV-1_0.1.6 demonstrates that cellPACK can keep up with the fast pace of HIV research. 


\section{Hierarchical cellPACK models}

The HIV-1 models presented here seek to characterize a single representative virion with full molecular detail. The hierarchical architecture of cellPACK allows this model to be used directly as an ingredient inside a larger environment recipe. Fig. 1B for example shows a rendering of HIV_in_Blood_Plasma_0.1.2, which incorporates HIV-1_0.1.4 as a complete ingredient packed amongst other plasma ingredients. Recipes can nest inside of other recipes in this manner, where a whole HIV particle is deposited as a single "large" ingredient according to its target density, or nested recipes can mix their ingredients to pack the HIV molecules at the same time as the blood plasma molecules, which allows depositing ingredients to weight and affect one another as they interact during packing. This specification of models with hierarchical recipes, rather than hierarchical models, offers great potential: In the process of building the model, nested recipes allow the ingredients in the different entities to interact and affect the final form of the model. For instance, antibodies in the blood plasma model can form interactions with the HIV model during creation of a larger model, which can restrict or induce other specific interactions based on densities and kinetics.

\section{Future plans}

We have initiated several projects to experiment with the HIV-cellPACK system, for example, to explore the vast ranges of interacting parameter space across all of the ingredients. An integrated fluorescence microscopy simulation tool, similar to the approach described by Gardner et al. ${ }^{76}$ was recently integrated into cellPACK to enable cellPACK models to be compared more directly to microscope images and to begin to test competing hypotheses for distribution of the Env glycoprotein in immature HIV-1 virions. Upcoming experiments will incorporate probabilities for specific interactions between known binding partners, and will fully explore the structures and interactions of host proteins as the research community identifies them. To further improve the HIV-1 mature virion model and to explore other aspects of the lifecycle, we have initiated a project to model the immature virion. Will exploring the vast parameter space of a cellPACK recipe in an automated fashion with results clustered to higher-order structural scoring functions enable cellPACK to reliably recapitulate the less well-described interaction details from better-understood neighboring molecular building blocks alone? These new approaches have begun to address this question.

Having refined the cellPACK software with the driving biological project of modeling HIV-1, it becomes a relatively short step to model a slightly larger and more complex organism such as Mycoplasma genitalium. A systems biology project called WholeCellviz has provided a computational recipe of the hypothetical quantities, localizations, and interactions of every gene product of M. genitalium, dynamically over a full life cycle. ${ }^{74}$ The WholeCellviz parameters are being transposed into cellPACK recipes that are completed by generating structural representations for each gene product. When complete, the recipe will be packed to generate the first 3D structural model of a whole cell and the first structural snapshots of a whole cell dynamically changing over an entire cell cycle. On a broader scale, because it collects Systems Biology and Structural Biology results into a unified framework, cellPACK can support big data projects like the 3D Virtual Cell project (http://3dvcell.org), where teams of biologists will merge 
efforts to construct virtual cells that can be probed experimentally to predict outcomes ranging from molecular mechanisms to cell-wide effects.

\section{Acknowledgements}

We would like to thank Megan Riel-Mehan for writing suggestions; Andrew McWhae for his award-winning image of HIV_in_Blood_Plasma_0.1.2; Thomas Goddard for efficient/coarse models of $3 \mathrm{j} 3 \mathrm{q}$ and HIV RNA; Barbie K. GanserPornillos, Owen Pornillos, Mark Yeager, Juan R. Perilla and Klaus Schulten for additional unpublished versions of their respective HIV capsid models. This work was supported in part by a predoctoral fellowship from NSF (NSF 07576), a grant from the NIH (P41 RR08605), a QB3 Fellowship grant from the California Institute for Quantitative Biosciences, qb3@UCSF, and a UCSF School of Pharmacy, 2013 Mary Anne Koda-Kimble Seed Award for Innovation. This is manuscript 27000 from the Scripps Research Institute.

\section{References}

1 B. K. Ganser-Pornillos, M. Yeager and W. I. Sundquist, Curr. Opin. Struct. Biol., 2008, 18, 203-217.

2 D. Lyumkis, J. P. Julien, N. de Val, A. Cupo, C. S. Potter, P. J. Klasse, D. R. Burton, R. W. Sanders, J. P. Moore, B. Carragher, I. A. Wilson and A. B. Ward, Science, 2013, 342(6165), 1484.

3 G. Zhao, J. R. Perilla, E. L. Yufenyuy, X. Meng, B. Chen, J. Ning, J. Ahn, A. M. Gronenborn, K. Schulten, C. Aiken and P. Zhang, Nature, 2013, 497, 643-646.

4 J. M. Grime and G. A. Voth, Biophys. J., 2012, 103, 1774-1783.

5 D. Russel, K. Lasker, B. Webb, J. Velazquez-Muriel, E. Tjioe, D. SchneidmanDuhovny, B. Peterson and A. Sali, PLoS Biol., 2012, 10, e1001244.

6 M. Blanco, J. Comput. Chem., 1991, 12, 237-247.

7 T. Byholm, M. Toivakka and J. Westerholm, Powder Technol., 2009, 196, 139146.

8 T. AndoJ. Skolnick, Proceedings of the International Conference of the Quantum Bio-Informatics IV., ed. L. Accardi, W. Freundenberg and M. Ohya, World Scientific Publishing Co., 2011, 28, pp. 413-426.

9 S. R. McGuffee and A. H. Elcock, PLoS Comput. Biol., 2010, 6, e1000694.

10 S. Takamori, M. Holt, K. Stenius, E. A. Lemke, M. Gronborg, D. Riedel, H. Urlaub, S. Schenck, B. Brugger, P. Ringler, S. A. Muller, B. Rammner, F. Grater, J. S. Hub, B. L. De Groot, G. Mieskes, Y. Moriyama, J. Klingauf, H. Grubmuller, J. Heuser, F. Wieland and R. Jahn, Cell, 2006, 127, 831-846.

11 D. S. Goodsell, Biochem. Mol. Biol. Educ., 2009, 37, 325-332.

12 D. S. Goodsell, Biochem. Mol. Biol. Educ., 2011, 39, 91-101.

13 D. S. Goodsell, http://www.pdb.org/pdb/education_discussion/ educational_resources/hiv-animation.html, RCSB Protein Data Bank, 2011.

14 B. K. Ganser-Pornillos, M. Yeager and O. Pornillos, Adv. Exp. Med. Biol., 2012, 726, 441-465.

15 J. A. Briggs and H. G. Krausslich, J. Mol. Biol., 2011, 410, 491-500.

16 W. I. Sundquist and H. G. Krausslich, Cold Spring Harbor Perspect. Med., 2012, 2, a006924. 
17 A. A. Waheed and E. O. Freed, AIDS Res. Hum. Retroviruses, 2012, 28, 54-75.

18 M. Balasubramaniam and E. O. Freed, Physiology (Bethesda), 2011, 26, 236251.

19 D. S. Goodsell, Biochem Mol Biol Educ, 2012, 40, 291-296.

20 M. F. Sanner, A. J. Olson and J. C. Spehner, Biopolymers, 1996, 38, 305-320.

21 G. T. Johnson, L. Autin, D. S. Goodsell, M. F. Sanner and A. J. Olson, Structure, 2011, 19, 293-303.

22 M. F. Sanner, J. Mol. Graph. Model., 1999, 17, 57-61.

23 E. F. Pettersen, T. D. Goddard, C. C. Huang, G. S. Couch, D. M. Greenblatt, E. C. Meng and T. E. Ferrin, J. Comput. Chem., 2004, 25, 1605-1612.

24 L. Autin, G. Johnson, J. Hake, A. Olson and M. Sanner, IEEE Comput. Graphics Appl., 2012, 32, 50-61.

25 J. A. Briggs, K. Grunewald, B. Glass, F. Forster, H. G. Krausslich and S. D. Fuller, Structure, 2006, 14, 15-20.

26 P. Zhu, J. Liu, J. Bess, Jr., E. Chertova, J. D. Lifson, H. Grise, G. A. Ofek, K. A. Taylor and K. H. Roux, Nature, 2006, 441, 847-852.

27 J. Chojnacki, T. Staudt, B. Glass, P. Bingen, J. Engelhardt, M. Anders, J. Schneider, B. Muller, S. W. Hell and H. G. Krausslich, Science, 2012, 338, 524-528.

28 T. Wilk, I. Gross, B. E. Gowen, T. Rutten, F. de Haas, R. Welker, H. G. Krausslich, P. Boulanger and S. D. Fuller, J. Virol., 2001, 75, 759-771.

29 J. A. Briggs, T. Wilk, R. Welker, H. G. Krausslich and S. D. Fuller, EMBOJ., 2003, 22, 1707-1715.

30 P. Zhu, E. Chertova, J. Bess, Jr., J. D. Lifson, L. O. Arthur, J. Liu, K. A. Taylor and K. H. Roux, Proc. Natl. Acad. Sci. U. S. A., 2003, 100, 15812-15817.

31 J. A. Briggs, M. N. Simon, I. Gross, H. G. Krausslich, S. D. Fuller, V. M. Vogt and M. C. Johnson, Nat. Struct. Mol. Biol., 2004, 11, 672-675.

32 L. A. Carlson, J. A. Briggs, B. Glass, J. D. Riches, M. N. Simon, M. C. Johnson, B. Muller, K. Grunewald and H. G. Krausslich, Cell Host Microbe, 2008, 4, 592599.

33 O. Pornillos, B. K. Ganser-Pornillos, B. N. Kelly, Y. Hua, F. G. Whitby, C. D. Stout, W. I. Sundquist, C. P. Hill and M. Yeager, Cell, 2009, 137, 12821292.

34 O. Pornillos, B. K. Ganser-Pornillos and M. Yeager, Nature, 2011, 469, 424-427.

35 R. Welker, H. Kottler, H. R. Kalbitzer and H. G. Krausslich, Virology, 1996, 219, 228-236.

36 T. Jacks, M. D. Power, F. R. Masiarz, P. A. Luciw, P. J. Barr and H. E. Varmus, Nature, 1988, 331, 280-283.

37 A. D. Frankel and J. A. Young, Annu. Rev. Biochem., 1998, 67, 1-25.

38 E. R. Wright, J. B. Schooler, H. J. Ding, C. Kieffer, C. Fillmore, W. I. Sundquist and G. J. Jensen, EMBO J., 2007, 26, 2218-2226.

39 N. Kol, M. Tsvitov, L. Hevroni, S. G. Wolf, H. B. Pang, M. S. Kay and I. Rousso, J. Virol. Methods, 2010, 169, 244-247.

40 J. S. Saad, J. Miller, J. Tai, A. Kim, R. H. Ghanam and M. F. Summers, Proc. Natl. Acad. Sci. U. S. A., 2006, 103, 11364-11369.

41 A. Alfadhli, R. L. Barklis and E. Barklis, Virology, 2009, 387, 466-472.

42 A. Alfadhli, D. Huseby, E. Kapit, D. Colman and E. Barklis, J. Virol., 2007, 81, 1472-1478. 
43 J. A. Briggs, M. C. Johnson, M. N. Simon, S. D. Fuller and V. M. Vogt, J. Mol. Biol., 2006, 355, 157-168.

44 S. C. Pettit, M. D. Moody, R. S. Wehbie, A. H. Kaplan, P. V. Nantermet, C. A. Klein and R. Swanstrom, J. Virol., 1994, 68, 8017-8027.

45 H. G. Krausslich, M. Facke, A. M. Heuser, J. Konvalinka and H. Zentgraf, J. Virol., 1995, 69, 3407-3419.

46 J. L. Newman, E. W. Butcher, D. T. Patel, Y. Mikhaylenko and M. F. Summers, Protein Sci., 2004, 13, 2101-2107.

47 M. A. Accola, S. Hoglund and H. G. Gottlinger, J. Virol., 1998, 72, 2072-2078.

48 I. P. O'Carroll, F. Soheilian, A. Kamata, K. Nagashima and A. Rein, Virus Res., 2013, 171, 341-345.

49 R. J. Fisher, A. Rein, M. Fivash, M. A. Urbaneja, J. R. Casas-Finet, M. Medaglia and L. E. Henderson, J. Virol., 1998, 72, 1902-1909.

50 A. de Marco, A. M. Heuser, B. Glass, H. G. Krausslich, B. Muller and J. A. Briggs, J. Virol., 2012, 86, 13708-13716.

51 S. M. Solbak, T. R. Reksten, F. Hahn, V. Wray, P. Henklein, P. Henklein, O. Halskau, U. Schubert and T. Fossen, Biochim. Biophys. Acta, Biomembr., 2013, 1828, 816-823.

52 T. Schwede, J. Kopp, N. Guex and M. C. Peitsch, Nucleic Acids Res., 2003, 31, 3381-3385.

53 D. Lyumkis, J. P. Julien, N. de Val, A. Cupo, C. S. Potter, P. J. Klasse, D. R. Burton, R. W. Sanders, J. P. Moore, B. Carragher, I. A. Wilson and A. B. Ward, Science, 2013, 342, 1484-1490.

54 J. Liu, A. Bartesaghi, M. J. Borgnia, G. Sapiro and S. Subramaniam, Nature, 2008, 455, 109-113.

55 H. R. Gelderblom, E. H. Hausmann, M. Ozel, G. Pauli and M. A. Koch, Virology, 1987, 156, 171-176.

56 E. Chertova, J. W. Bess, Jr., B. J. Crise, I. R. Sowder, T. M. Schaden, J. M. Hilburn, J. A. Hoxie, R. E. Benveniste, J. D. Lifson, L. E. Henderson and L. O. Arthur, J. Virol., 2002, 76, 5315-5325.

57 S. Lopez-Verges, G. Camus, G. Blot, R. Beauvoir, R. Benarous and C. BerliozTorrent, Proc. Natl. Acad. Sci. U. S. A., 2006, 103, 14947-14952.

58 M. A. Checkley, B. G. Luttge, P. Y. Mercredi, S. K. Kyere, J. Donlan, T. Murakami, M. F. Summers, S. Cocklin and E. O. Freed, J. Virol., 2013, 87, 3561-3570.

59 M. A. Checkley, B. G. Luttge and E. O. Freed, J. Mol. Biol., 2011, 410, 582-608.

60 M. Kogan and J. Rappaport, Retrovirology, 2011, 8, 25.

61 B. Muller, U. Tessmer, U. Schubert and H. G. Krausslich, J. Virol., 2000, 74, 9727-9731.

62 X. Ren, S. Y. Park, J. S. Bonifacino and J. H. Hurley, eLife, 2014, 3, e01754.

63 N. Eswar, B. Webb, M. A. Marti-Renom, M. S. Madhusudhan, D. Eramian, M. Y. Shen, U. Pieper and A. Sali, Current protocols in bioinformatics / editoral board, Andreas D. Baxevanis... [et al.], 2006, Chapter 5, Unit 56.

64 M. W. Pandori, N. J. Fitch, H. M. Craig, D. D. Richman, C. A. Spina and J. C. Guatelli, J. Virol., 1996, 70, 4283-4290.

65 Y. Guo, L. Dong, X. Qiu, Y. Wang, B. Zhang, H. Liu, Y. Yu, Y. Zang, M. Yang and Z. Huang, Nature, 2014, 505, 229-233. 
66 L. Ratner, W. Haseltine, R. Patarca, K. J. Livak, B. Starcich, S. F. Josephs, E. R. Doran, J. A. Rafalski, E. A. Whitehorn, K. Baumeister, et al., Nature, 1985, 313, 277-284.

67 N. Jouvenet, S. Laine, L. Pessel-Vivares and M. Mougel, RNA Biol., 2011, 8, 572580.

68 J. M. Watts, K. K. Dang, R. J. Gorelick, C. W. Leonard, J. W. Bess, Jr., R. Swanstrom, C. L. Burch and K. M. Weeks, Nature, 2009, 460, 711-716.

69 D. E. Ott, Rev. Med. Virol., 2008, 18, 159-175.

70 E. K. Franke, H. E. Yuan and J. Luban, Nature, 1994, 372, 359-362.

71 J. E. Dooher, B. L. Schneider, J. C. Reed and J. R. Lingappa, Traffic, 2007, 8, 195-211.

72 L. Chatel-Chaix, K. Boulay, A. J. Mouland and L. Desgroseillers, Retrovirology, 2008, 5, 41.

73 E. V. Ryzhova, R. M. Vos, A. V. Albright, A. V. Harrist, T. Harvey and F. Gonzalez-Scarano, J. Virol., 2006, 80, 2694-2704.

74 J. R. Karr, J. C. Sanghvi, D. N. Macklin, M. V. Gutschow, J. M. Jacobs, B. Bolival, Jr., N. Assad-Garcia, J. I. Glass and M. W. Covert, Cell, 2012, 150, 389-401.

75 B. G. Wilhelm, S. Mandad, S. Truckenbrodt, K. Krohnert, C. Schafer, B. Rammner, S. J. Koo, G. A. Classen, M. Krauss, V. Haucke, H. Urlaub and S. O. Rizzoli, Science, 2014, 344, 1023-1028.

76 M. K. Gardner, D. J. Odde and K. Bloom, Methods, 2007, 41, 232-237. 